Pacific Journal of Mathematics

RNAG WTIT BOVNDED ANYHLA ATO 


\title{
ON RINGS WITH BOUNDED ANNIHILATORS
}

\author{
John A. Beachy and William-D. Blair
}

\begin{abstract}
Commutative rings for which the annihilator of each finitely generated module is determined by $n$ or fewer elements are studied. For Artinian rings the bound $n$ is explicitly determined. Noetherian rings satisfying this condition are of Krull dimension one. A new characterization of Dedekind domains is given, and the relationship between the bound and the number of generators of ideals is studied.
\end{abstract}

It is well-known that for commutative Noetherian rings there is a one-to-one correspondence between prime ideals and isomorphism classes of indecomposable, injective modules. Gabriel [6] showed that this correspondence still holds for any noncommutative Noetherian ring $R$ which satisfies the following condition $(\mathrm{H})$ : for each finitely generated module ${ }_{R} M$ there exist elements $m_{1}, m_{2}, \cdots, m_{n} \in M$ such that $\operatorname{Ann}_{R}(M)=\operatorname{Ann}_{R}\left(m_{1}, m_{2}, \cdots, m_{n}\right)$. (Here $\operatorname{Ann}_{R}(S)=\{r \in$ $R \mid r s=0$ for all $s \in S\}$, for any subset $S \subseteq M$.) Cauchon [4] showed that the converse holds, and thus condition $(\mathrm{H})$ holds, for example, for any noncommutative Noetherian ring which satisfies a polynomial identity or is integral over its center.

If the module ${ }_{R} M$ has the descending chain condition on annihilators $\operatorname{Ann}(S)$ of subsets $S \subseteq M$, then it is clear that $\operatorname{Ann}_{R}(M)=$ $\operatorname{Ann}_{R}\left(m_{1}, m_{2}, \cdots, m_{n}\right)$ for elements $m_{1}, m_{2}, \cdots, m_{n} \in M$. Thus the class of left Artinian rings is one of the basic classes for which Gabriel's condition $(\mathrm{H})$ holds. In fact, the ring $R$ is left Artinian if and only if the condition on annihilators holds for all left $R$-modules, not just the finitely generated modules (see [2]). If $R$ is left Artinian, we note that the number of elements required in condition $(\mathrm{H})$ is bounded by the length of a composition series for the ring $R$. This motivates the following definition.

Definition. An $R$-module $M$ is said to have bound $n$ if $n$ is the least integer such that $\operatorname{Ann}_{R}(M)=\operatorname{Ann}_{R}\left\{m_{1}, \cdots, m_{n}\right\}$ for some finite subset $\left\{m_{1}, \cdots, m_{n}\right\} \subseteq M$. A ring $R$ is said to have (global) bound $n$, denoted by $b d(R)=n$, if $n$ is the supremum of the bounds of all finitely generated $R$-modules.

We note that an $R$-module $M$ has bound $n$ if and only if $n$ is the least integer such that there exists an exact sequence $0 \rightarrow$ $R / \operatorname{Ann}_{R}(M) \rightarrow M^{n}$, where $M^{n}$ denotes the direct sum of $n$ copies of $M$. This fact will be used repeatedly in the paper. 
We have been able to compute the bound of a commutative Artinian ring in terms of the lengths of homogeneous components of the socles of factor rings, but a similar characterization for noncommutative Artinian rings appears to be much more difficult to obtain. In this paper we restrict our study to commutative Noetherian rings, in which case the bound of a ring can be related to the number of generators required for ideals of the ring. We note, however, that a ring with a finite bound need not be Noetherian. In fact, if $R$ is commutative and the module ${ }_{R} M$ is a direct sum $\bigoplus_{\alpha \in I} R m_{\alpha}$ of cyclic submodules $R m_{\alpha}$, then $A_{n} n_{R}(M)=\operatorname{Ann}_{R}(m)$ for the element $m=\left(m_{\alpha}\right)_{\alpha \in I} \in M$. Thus any commutative ring whose finitely generated modules are direct sums of cyclic modules has bound 1, and such rings need not be Noetherian (see Brandal [3]).

We show that a commutative Noetherian ring with a finite bound must have Krull dimension 1. In this case, the ring has bound 1 if and only if it is a ZPI-ring, and so a Noetherian domain is a Dedekind domain if and only if it has bound 1. For a local Noetherian domain, the existence of a finite bound is equivalent to the existence of a bound on the number of generators required for any ideal of the ring, and in this case the two numbers are equal. For a nonlocal domain, the two numbers differ by at most one. In the last part of the paper we consider the problem for rings other than domains by using a weaker notion of bound.

All rings under consideration will be assumed to be commutative rings with unity, and all modules will be assumed to be unital. The injective hull of a module ${ }_{R} M$ in the category of $R$-modules will be denoted by $E_{R}(M)$.

We will make extensive use of the notion of the Goldie dimension of a module. (The reader is referred to Goldie [7] for details.) A nonzero module is said to be uniform if any two nonzero submodules have a nonzero intersection. A submodule $N$ of $M$ is essential in $M$ if $N$ nontrivially intersects any nonzero submodule of $M$. A module $M$ has Goldie (or uniform) dimension $n$ if $M$ contains a direct sum of $n$ nonzero submodules, but no longer direct sum. Then $M$ has Goldie dimension $n$ if and only if $M$ contains an essential direct sum of $n$ uniform submodules, or equivalently, if and only if $E(M)$ is a direct sum of $n$ indecomposable submodules. It is evident that the Goldie dimension of a submodule of $M$ is less than or equal to that of $M$.

We begin with an easy but useful lemma.

Lemma 1. (i) Let $M$ be an $R$-module. If the Goldie dimension of $R$ is $n$ and $0 \rightarrow R \rightarrow M^{t}$ is an exact sequence, then there is a homomorphism such that $0 \rightarrow R \rightarrow M^{n}$ is exact. 
(ii) If $b d(R)=n$, then $b d(R / I) \leqq n$ for each ideal $I$ of $R$.

(iii) $b d\left(R_{1} \oplus \cdots \oplus R_{s}\right)=\max _{1 \leqq i \leqq s}\left\{b d\left(R_{i}\right)\right\}$.

(iv) Let $S$ be a multiplicative set. Then $b d\left(R_{S}\right) \leqq b d(R)$.

Proof. ( i ): Let $\left(m_{1}, \cdots, m_{t}\right)$ be the image of 1 under $R \rightarrow M^{t}$, and then note that $\left\{I_{j}\right\}, j=1, \cdots, t$ is a direct family where $I_{j}=$ $\bigcap_{i \neq j} \operatorname{Ann}_{R}\left\{m_{i}\right\}$.

(ii) and (iii) are clear.

(iv): Let $M$ be an $R_{s}$-module generated by $x_{1}, \cdots, x_{t}$. Considering $M$ as an $R$-module, let $N$ be the $R$-submodule generated by $x_{1}, \cdots, x_{t}$. Then there exist $m_{1}, \cdots, m_{n} \in N$ such that $\operatorname{Ann}_{R}\left\{m_{1}, \cdots\right.$, $\left.m_{n}\right\}=\operatorname{Ann}_{R}(N)$, where $n=b d(R) . \operatorname{But}_{A_{n}}\left\{m_{1}, \cdots, m_{n}\right\}=\operatorname{Ann}_{R_{S}}(M)$ and so $b d\left(R_{S}\right) \leqq n$.

Recall that the socle, $\operatorname{Soc}(M)$, of a module $M$ is the sum of its minimal $(\neq 0)$ submodules. The socle is a direct sum of certain of the minimal submodules. A homogeneous component of the socle is the sum of all minimal submodules in an isomorphism class of minimal submodules.

THeORem 2. Let $R$ be an Artinian ring. The bound of $R$ is the supremum of the lengths of the homogeneous components of the socle of $R / A$, for all ideals $A$ of $R$.

Proof. Observe that the supremum exists since the length of $R / A$ is at most the length of $R$ for all ideals $A$ of $R$.

Let $n$ be the supremum of the lengths of the homogeneous components of the socles of all factor rings of $R$. We first show that $b d(R) \geqq n$. Suppose that $\bar{R}=R / A$ has a homogeneous component of its socle of length $n$, and let $I$ be a minimal ideal of $\bar{R}$ which appears as a direct summand of the socle $S$ of $\bar{R}$ exactly $n$ times. Let $X$ be a direct sum of one minimal ideal from each isomorphism class of minimal ideals of $\bar{R}$. We consider the injective hull $E=$ $E_{\bar{R}}(X)$ and show that $0 \rightarrow \bar{R} \rightarrow E^{n}$ is exact and that $n$ is the least number of copies of $E$ into which $\bar{R}$ can be embedded.

Since each homogeneous component of $S$ has at most $n$ summands, there exists an exact sequence $0 \rightarrow S \rightarrow E^{n}$. Since $E$ is injective and since $S$ is essential in $\bar{R}$, this extends to an exact sequence $0 \rightarrow E(\bar{R}) \rightarrow E^{n}$, and hence $\bar{R}$ embeds into $E^{n}$. If $0 \rightarrow \bar{R} \rightarrow E^{m}$ is exact for some $m<n$, we have that $E^{m}$ contains a direct sum of $n$ copies of $I$ and thus $X^{m}=\operatorname{Soc}\left(X^{m}\right)=\operatorname{Soc}\left(E^{m}\right)$ would contain $I^{n}$, a contradiction to the Krull-Schmidt theorem.

Let $\left(x_{1}, \cdots, x_{n}\right)$ be the image of $1_{\bar{R}}$ in $E^{n}$, and let $M$ be the $\bar{R}$ submodule of $E$ generated by $\left\{x_{1}, \cdots, x_{n}\right\}$. Then $M$ is a faithful, 
finitely generated $\bar{R}$-module such that $\bar{R}$ embeds into $n$ but no fewer copies of $M$. Hence $b d(R) \geqq b d(\bar{R}) \geqq n$. (We remark that it is not necessary to pass to $M$ since $X$ is a finite sum of minimal ideals, $E$ is a finite direct sum of indecomposable, injective $\bar{R}$-modules and hence is itself finitely generated by a theorem of Matlis.)

To show that $b d(R) \leqq n$, we note that $R=R_{1} \oplus \cdots \oplus R_{t}$ is a direct sum of local Artinian rings and since $b d(R)=\max \left\{b d\left(R_{i}\right)\right\}$, it suffices to show that $b d\left(R_{i}\right) \leqq n$ where $n$ is defined as above. Since each homogeneous component of $\operatorname{Soc}(R / A)$ is contained in some $R_{i} / A R_{i}$, it suffices to show that $b d(R) \leqq n$ when $R$ is a local Artinian ring.

Let $M$ be a finitely generated $R$-module and $\bar{R}=R / \mathrm{Ann}_{R}(M)$. Since $\bar{R}$ is local, all minimal ideals are isomorphic and the socle of $\bar{R}$ is homogeneous. Since every nonzero ideal contains a minimal ideal, $\operatorname{Soc}(\bar{R})$ is an essential direct sum of isomorphic minimal ideals, and thus the Goldie dimension of $\bar{R}$ is equal to the length of $\operatorname{Soc}(\bar{R})$. Since we have an embedding $0 \rightarrow \bar{R} \rightarrow M^{t}$ where $t$ is the length of $R$, there exists an exact sequence $0 \rightarrow \bar{R} \rightarrow M^{k}$ where $k$ is the length of $\operatorname{Soc}(\bar{R})$ by Lemma 1 . Since $k \leqq n$, there is an exact sequence $0 \rightarrow \bar{R} \rightarrow M^{n}$ and $b d(R) \leqq n$.

The following corollary will be useful in our study of Noetherian rings with a finite bound.

CoRollary 3. Let $R$ be a local Noetherian ring with maximal ideal $\mathfrak{m}$. If $\operatorname{dim}_{R / \mathrm{m}}\left(\mathfrak{m}^{k} / \mathfrak{m}^{k+1}\right)=n$ for some $k \in \boldsymbol{Z}^{+}$, then $b d(R) \geqq n$.

Proof. Since $R / \mathrm{m}^{k+1}$ is Artinian and since the (homogeneous) length of the socle of $R / \mathrm{m}^{k+1}$ is a least $n$, we have $b d(R) \geqq n$ by Theorem 2 .

Our next result shows how severe a restriction the existence of a bound places on the class of Noetherian rings.

THEOREM 4. Let $R$ be a Noetherian ring. If $R$ has finite bound, then the Krull dimension of $R$ is at most one.

Proof. Since the condition that $R$ has a finite bound passes to all localizations, we may assume that $R$ is a local ring. Let $\mathfrak{m}$ denote the maximal ideal of $R$ and let $b d(R)=n$. By Corollary 3, $\operatorname{dim}_{R / \mathfrak{m}}\left(\mathfrak{m}^{k} / \mathfrak{m}^{k+1}\right) \leqq n$ for all $k \in Z^{+}$. Let $f(k)$ be the length of $R / \mathfrak{m}^{k}$. For sufficiently large $k, f(k)$ is a polynomial in $k$ (Hilbert-Samuel theorem). The degree of $f(k)$ is less than or equal to 1 since $f(k+1)-f(k)$ is the length of $\mathrm{m}^{k} / \mathrm{m}^{k+1}$, which is bounded by $n$. By Theorem 11.14 of Atiyah-MacDonald [1], we have that the Krull dimension of $R$ is at most 1 . 
A ring $R$ is called a ZPI-ring (Zerlegung Primideale) if every ideal can be written as a product of prime ideals of $R . R$ is a ZPI-ring if and only if $R$ is a Noetherian ring such that for each maximal ideal $\mathfrak{m}$ of $R$ there are no ideals of $R$ strictly between $\mathfrak{m}$ and $\mathfrak{m}^{2}$. (See Larsen and McCarthy [9].)

THEOREM 5. $R$ is a Noetherian ring with $b d(R)=1$ if and only if $R$ is a ZPI-ring.

Proof. Let $R$ be a Noetherian ring with $b d(R)=1$ and let $m$ be a maximal ideal of $R$. Then $b d\left(R_{\mathrm{m}}\right)=1$ and Corollary 3 implies $\operatorname{dim}_{R_{\mathfrak{m}} / \mathrm{m} R_{\mathrm{m}}}\left(\mathfrak{m} R_{\mathrm{m}} / \mathfrak{m}^{2} R_{\mathrm{m}}\right) \leqq 1$. Thus there are no ideals of $R_{\mathrm{m}}$ strictly between $\mathfrak{m} R_{\mathrm{m}}$ and $\mathfrak{m}^{2} R_{\mathrm{m}}$, and so there are no ideals of $R$ between $\mathfrak{m}$ and $\mathfrak{m}^{2}$, since such ideals are $\mathfrak{m}$-primary.

Conversely, by Theorem 9.10 of Larsen and McCarthy [9], if $R$ is a ZPI-ring, then $R$ is a finite direct sum of Dedekind domains and special primary rings. Since any finitely generated module $M$ over a Dedekind domain $D$ is of the form $M \cong D / I_{1} \oplus \cdots \oplus D / I_{t}$ where $I_{1} \geqq I_{2} \geqq \cdots \geqq I_{t}$, we have that $I_{t}=\operatorname{Ann}_{D}(M)$ and thus there is an embedding of $D / \operatorname{Ann}_{D}(M)$ into $M$. Hence $b d(D)=1$. If $S$ is a special primary ring, then the socle of $S / I$ for any ideal $I$ has length 1 and thus by Theorem $2, b d(S)=1$. Hence $b d(R)=1$.

By Theorem 4 of Warfield [10], $R$ is a Noetherian ring with $b d(R)=1$ if and only if every finitely generated $R$ module is a direct summand of a direct sum of cyclic $R$-modules. We also point out that a special case of Theorem 5 gives a new characterization of Dedekind domains.

COROLlary 6. $R$ is a Dedekind domain if and only if $R$ is a Noetherian domain and $b d(R)=1$.

It is well known that every ideal in a Dedekind domain can be generated by two elements. As an extension of this last corollary we explore the relationship between Noetherian domains of finite bound and the number of elements required to generate their ideals. I. S. Cohen [5] designates the rank of a ring to be $n$ if every ideal of $R$ can be generated by $n$ or fewer elements. According to this definition a ring of rank $k$ is also of rank $h$ for all $h \geqq k$. We will say that $R$ has least rank $n$, denoted by $r k(R)=n$, if it has rank $n$ but not rank $n-1$.

THEOREM 7. Let $R$ be an Artinian ring. Then $b d(R)=r k(R)$. 
Proof. Suppose that $r k(R)=n$. Then $R=R_{1} \oplus \cdots \oplus R_{t}$ is a direct sum of local Artinian rings and $r k\left(R_{i}\right) \leqq n$. Let $A$ be an ideal of $R_{i}$. Then the socle of $R_{i} / A$ can be generated by $n$ or fewer elements and it is homogeneous, so the length of the socle is at most $n$. Hence $b d\left(R_{i}\right) \leqq n$ by Theorem 2 and $b d(R) \leqq n$.

Conversely, suppose that $b d(R)=n$. Then $R=R_{1} \oplus \cdots \oplus R_{t}$ is a direct sum of local Artinian rings with maximal ideals $\mathfrak{m}_{i}$ and $b d\left(R_{i}\right) \leqq n$. Let $I$ be an ideal of $R_{i}$ which requires $k$ generators. Thus $I / \mathfrak{m}_{i} I$ is a $k$-dimensional vector space over $R / \mathfrak{m}_{i}$. Hence the ring $R / \mathfrak{m}_{i} I$ has Goldie dimension at least $k$, and so the homogeneous length of the socle of $R / \mathfrak{m}_{i} I$ is at least $k$. Hence $b d\left(R_{i}\right) \geqq k$, and so $b d(R) \geqq k$. Thus $n=b d(R) \geqq r k(R)$, and the theorem is proved.

Theorem 8. Let $R$ be a Noetherian domain. Then $R$ has finite rank if and only if $R$ has finite bound, and in this case rk $(R)=$ $b d(R)$ or $r k(R)=b d(R)+1$. If $R$ is a local Noetherian domain, then $\operatorname{rk}(R)=b d(R)$.

Proof. If $R$ has finite rank or finite bound, then the Krull dimension of $R$ is one by Theorem 9 of Cohen [5] and Theorem 4 . Thus all proper factor rings of $R$ are Artinian.

Suppose that $r k(R)=n$. Let $M$ be a finitely generated $R$-module. If $\operatorname{Ann}_{R}(M)=0$, then since $R$ is a domain we have an exact sequence $0 \rightarrow R \rightarrow M$. If $\operatorname{Ann}(M) \neq 0$, then $R / \operatorname{Ann}_{R}(M)$ is an Artinian ring and since $r k\left(R / \operatorname{Ann}_{R}(M)\right)=b d\left(R / \operatorname{Ann}_{R}(M)\right) \leqq n, R / \mathrm{Ann}_{R}(M) \mathrm{em}$ beds into $n$ copies of $M$. In any case, there is an exact sequence $0 \rightarrow R / \operatorname{Ann}_{R}(M) \rightarrow M^{n}$ and so $b d(R) \leqq r k(R)$.

Suppose that $b d(R)=n$. If $I$ is a nonzero ideal of $R$, then choose $0 \neq a \in I$. Then $R / a R$ is Artinian and $b d(R / a R) \leqq n$. Thus $r k(R / a R) \leqq n$ and $I$ can be generated by $n+1$ (or fewer) elements. Thus $r k(R) \leqq b d(R)+1$.

Now suppose that $R$ is a local Noetherian domain with maximal ideal $\mathrm{m}$ and $b d(R)=n$. If $I$ is an ideal of $R$ requiring $k$ generators, then $\operatorname{dim}_{R / \mathfrak{m}}(I / I \mathfrak{m})=k$. Thus the Goldie dimension of $R / I \mathfrak{m}$ is at least $k$ and so the homogeneous length of the socle of $R / I \mathrm{~m}$ is at least $k$. Since $R / I \mathfrak{m}$ is Artinian, $b d(R / I \mathfrak{m}) \geqq k$ and thus $n \geqq k$ and $b d(R) \geqq r k(R)$.

Corollary 9. Let $R$ be a local Noetherian domain. Then $R$ has finite bound if and only if the Krull dimension of $R$ is at most one.

Proposition 10. Let $R$ be a Noetherian domain. If $b d\left(R_{\mathfrak{m}}\right) \leqq n$ for all maximal ideals $\mathfrak{m}$ of $R$, then $b d(R) \leqq n$. 
Proof. We first observe that if $R_{\mathrm{m}}$ has finite bound for all maximal ideals $\mathfrak{m}$, then $R$ has Krull dimension at most one. Let $M$ be a finitely generated $R$-module, with $A=\operatorname{Ann}_{R}(M)$. If $A=0$, then there exists an embedding $0 \rightarrow R \rightarrow M$. If $A \neq 0$, then $R / A$ is Artinian and $R / A \cong R_{\mathfrak{p}_{1}} / \mathfrak{q}_{1} R_{\mathfrak{p}_{1}} \oplus \cdots \oplus R_{p_{t}} / \mathfrak{q}_{t} R_{\mathrm{p}_{t}}$ where $A=\mathfrak{q}_{1} \cap \cdots \cap \mathfrak{q}_{t}$ is a primary decomposition of $A$ and each $\mathfrak{q}_{i}$ is $\mathfrak{p}_{i}$-primary. Thus there exists an embedding $0 \rightarrow R / A \rightarrow M^{n}$ since $b d\left(R_{\mathfrak{p}_{i}}\right) \leqq n$ for each $i$. In any case, $R / A$ embeds into $n$ copies of $M$, and hence $b d(R) \leqq n$.

Recall that if $R$ is a domain with quotient field $K$, then any subring $S$ with $R \subseteq S \subseteq K$ is called an overring of $R$. The following theorem extends the well-known result that any overring of a Dedekind domain is a Dedekind domain.

Theorem 11. Let $R$ be a Noetherian domain with finite bound, and let $K$ be the quotient field of $R$. If $S$ is an overring of $R$, then $b d(S) \leqq b d(R)$.

Proof. By Proposition 10 it is sufficient to show that $b d\left(S_{\mathrm{m}}\right) \leqq$ $b d(R)$ for all maximal ideals of $S$. Let $m$ be a maximal ideal of $S$ and let $\mathfrak{m}^{\prime}=\mathfrak{m} \cap R$. Then $S_{\mathrm{m}}$ is an overring of $R_{\mathrm{m}^{\prime}}$ and both rings have Krull dimension at most one by Theorem 93 of Kaplansky [8]. The proof of that theorem shows that if $A$ is a nonzero ideal of $S_{\mathrm{m}}$, then $S_{\mathrm{m}} / A$ can be embedded in a proper cyclic $R_{\mathrm{m}}$-module, and so the Goldie dimension of $S_{\mathrm{m}} / A$ is at most that of the cyclic $R_{\mathrm{m}^{\prime}}$-module. Since $S_{\mathrm{m}}$ and $R_{\mathrm{m}^{\prime}}$ are local, the socle of any proper factor ring is homogeneous, and so the length of the socle of $S_{\mathrm{m}} / A$ is at most $b d\left(R_{\mathrm{m}^{\prime}}\right) \leqq b d(R)$ and hence $b d\left(S_{\mathrm{m}}\right) \leqq b d(R)$.

Next we wish to consider Noetherian rings which are not necessarily domains. For this purpose we introduce the notion of a weak bound.

Definition. The ring $R$ has weak bound at most $n$, denoted $w b d(R) \leqq n$, if for every finitely generated $R$-module $M$ such that $\operatorname{Ann}_{R}(M)$ has no embedded primes there exist $n$ elements $m_{1}, \cdots$, $m_{n} \in M$ such that $\operatorname{Ann}_{R}(M)=\operatorname{Ann}_{R}\left\{m_{1}, \cdots, m_{n}\right\}$. If $w b d(R) \leqq n$ but $w b d(R) \nless n$, then we write $w b d(R)=n$.

Proposition 12. If $R$ is a Noetherian ring and $w b d(R) \leqq n$, then the Krull dimension of $R$ is at most one.

Proof. We first observe that if $S$ is a multiplicative set in $R$, 
then $w b d(R) \leqq n$ implies $w b d\left(R_{S}\right) \leqq n$. To see this let $M$ be a finitely generated $R_{S}$-module whose annihilator has no embedded primes. If $\left\{x_{1}, \cdots, x_{t}\right\}$ is a set of generators of $M$, then as in the proof of Lemma 1 let $N$ be the $R$-submodule of $M$ generated by $x_{1}, \cdots, x_{t}$. Then $\operatorname{Ann}_{R}(N)=\operatorname{Ann}_{R_{S}}(M) \cap R$ has no embedded primes, and so there exist $m_{1}, \cdots, m_{n} \in N$ such that $\operatorname{Ann}_{R}(N)=\operatorname{Ann}_{R}\left\{m_{1}, \cdots\right.$, $\left.m_{n}\right\}$. Thus $\operatorname{Ann}_{R_{S}}(M)=\operatorname{Ann}_{R_{S}}\left\{m_{1}, \cdots, m_{n}\right\}$.

The proof of the proposition follows from Corollary 3 exactly as in the proof of Theorem 4 because $w b d(R)=b d(R)$ for Artinian rings.

CoRollaRy 13. If $R$ is a Noetherian domain, then $w b d(R)=$ $b d(R)$.

Lemma 14. Let $\mathfrak{p}$ be a prime ideal of the Noetherian ring $R$, and suppose that $R / \mathfrak{p}$ has finite rank. Then $R / \mathfrak{p}^{k}$ has finite rank for each $k \in Z^{+}$.

Proof. The proof is by induction on $k$. The case $k=1$ is true by hypothesis. Assume that $R / p^{k-1}$ has finite rank. Thus every $R$-submodule of $R / \mathfrak{p}^{k-1}$ can be generated by $r k\left(R / \mathfrak{p}^{k-1}\right)$ elements. The $R / \mathfrak{p}$-module $\mathfrak{p}^{k-1} / \mathfrak{p}^{k}$ is generated by say, $m$ elements. Thus any submodule of $\mathfrak{p}^{k-1} / \mathfrak{p}^{k}$ can be generated by $m \cdot r k(R / \mathfrak{p})$ elements. Hence any ideal of $R / \mathfrak{p}^{k}$ can be generated by $r k\left(R / \mathfrak{p}^{k-1}\right)+m \cdot r k(R / \mathfrak{p})$ elements and thus $R / p^{k}$ has finite rank.

THEOREM 15. Let $R$ be a commutative Noetherian ring. Then $R$ has finite rank if and only if $R$ has finite weak bound.

Proof. Suppose that $r k(R)=n$ and suppose that $R$ has $t$ minimal prime ideals. Let $M$ be a finitely generated $R$-module whose annihilator has no embedded primes. Let $\operatorname{Ann}_{R}(M)=\mathfrak{q}_{1} \cap \cdots \cap \mathfrak{q}_{s} \cap$ $\mathfrak{q}_{s+1} \cap \cdots \cap \mathfrak{q}_{k}$ be a primary decomposition of $A \mathrm{nn}_{R}(M)$, where $\mathfrak{q}_{i}$ is $\mathfrak{p}_{i}$-primary and $\mathfrak{p}_{1}, \cdots, \mathfrak{p}_{s}$ are minimal prime ideals of $R$ while $\mathfrak{p}_{s+1}, \cdots, \mathfrak{p}_{k}$ are maximal ideals of $R$. Since $R$ has Krull dimension at most one and $\operatorname{Ann}_{R}(M)$ has no embedded primes, we have that $\mathfrak{q}_{1} \cap \cdots \cap \mathfrak{q}_{s}$ and $\mathfrak{q}_{s+1} \cap \cdots \cap \mathfrak{q}_{k}$ are comaximal ideals for if $\mathfrak{m}$ is a maximal ideal containing $\mathfrak{q}_{i} \cap \cdots \cap \mathfrak{q}_{s}$ and $\mathfrak{q}_{s+1} \cap \cdots \cap \mathfrak{q}_{k}$, then $\mathfrak{m}$ contains some $\mathfrak{q}_{i}$ with $1 \leqq i \leqq s$ and some $\mathfrak{q}_{j}$ with $s+1 \leqq j \leqq k$ and so $\mathfrak{m}=\mathfrak{p}_{j} \supseteqq \mathfrak{p}_{i}$ contradicting the fact that $\operatorname{Ann}_{R}(M)$ has no embedded primes. Thus

$$
R / \operatorname{Ann}_{R}(M) \cong R /\left(\mathfrak{q}_{1} \cap \cdots \cap \mathfrak{q}_{s}\right) \oplus R /\left(\mathfrak{q}_{s+1} \cap \cdots \cap \mathfrak{q}_{k}\right) .
$$

Since $R /\left(\mathfrak{q}_{s+1} \cap \cdots \cap \mathfrak{q}_{k}\right) \cong R / \mathfrak{q}_{s+1} \oplus \cdots \oplus R / \mathfrak{q}_{k}$ is Artinian and since $r k(R)=n$, we have that the bound and hence the weak bound of 
$R /\left(\mathfrak{q}_{s+1} \cap \cdots \cap \mathfrak{q}_{k}\right)$ is at most $n$.

On the other hand, since for $i=1, \cdots, s, \mathfrak{q}_{i}=\mathfrak{q}_{i} R_{\mathrm{p}_{i}} \cap R$, we have that $R / \mathfrak{q}_{i}$ embeds into $R_{\mathfrak{p}_{i}} / \mathfrak{q}_{i} R_{p_{i}}$ where $R_{p_{i}}$ is the Artinian local ring obtained by localizing $R$ at $\mathfrak{p}_{i}$. Since $r k\left(R_{p_{i}}\right) \leqq n$, the Goldie dimension of any factor ring of $R_{p_{i}}$ is at most $n$. Noting that the Goldie dimension of $R_{p_{i}} / \mathfrak{q}_{i} R_{p_{i}}$ as a ring is the same as the Goldie dimension of $R_{p_{i}} / \mathfrak{q}_{i} R_{p_{i}}$ considered as an $R_{p_{i}}$-module, which is the same as the Goldie dimension when it is considered as an $R$-module, we see that the Goldie dimension of $R / \mathfrak{q}_{i}$ as an $R$-module is at most $n$. Since $s \leqq t$ and since $R /\left(\mathfrak{q}_{1} \cap \cdots \cap \mathfrak{q}_{s}\right)$ is contained in $R / \mathfrak{q}_{1} \oplus \cdots \oplus R / \mathfrak{q}_{s}$, we see that the ring $R /\left(\mathfrak{q}_{1} \cap \cdots \cap \mathfrak{q}_{s}\right)$ has Goldie dimension at most $t \cdot n$. Thus $R /\left(\mathfrak{q}_{1} \cap \cdots \cap \mathfrak{q}_{s}\right)$ embeds into $n t$ copies of any faithful module over it. Therefore $R / \mathrm{Ann}_{R}(M)$ embeds into $n t$ copies of $M$, since $M=\left(R /\left(\mathfrak{q}_{1} \cap \cdots \cap \mathfrak{q}_{s}\right)\right) M \oplus\left(R /\left(\mathfrak{q}_{s+1} \cap \cdots \cap \mathfrak{q}_{k}\right)\right) M$ and $\left(R /\left(\mathfrak{q}_{1} \cap \cdots \cap \mathfrak{q}_{s}\right)\right) M$ is a faithful $R /\left(\mathfrak{q}_{1} \cap \cdots \cap \mathfrak{q}_{s}\right)$-module and $\left(R /\left(\mathfrak{q}_{s+1} \cap \cdots \cap \mathfrak{q}_{k}\right)\right) M$ is a faithful $R /\left(\mathfrak{q}_{s+1} \cap \cdots \cap \mathfrak{q}_{k}\right)$-module. Thus $w b d(R) \leqq n t$.

Conversely, suppose that $w b d(R)=n$. Then for each prime ideal $\mathfrak{p}$ of $R, R / \mathfrak{p}$ is a Noetherian domain and $b d(R / \mathfrak{p})=w b d(R / \mathfrak{p}) \leqq n$. Thus $r k\left(R / \mathfrak{p}^{k}\right) \leqq n+1$. By Lemma $14, r k\left(R / \mathfrak{p}^{k}\right)<\infty$ for each $k \in Z^{+}$.

From a primary decomposition of 0 we see that there exists an integer $k$ such that $0=\bigcap_{i=1}^{t} \mathfrak{p}_{i}^{k}$ where the $\mathfrak{p}_{i}$ are the minimal primes of $R$. Hence we have an exact sequence

$$
0 \longrightarrow R \longrightarrow R / \mathfrak{p}_{1}^{k} \oplus \cdots \oplus R / \mathfrak{p}_{t}^{k}
$$

Since $r k\left(R / \mathfrak{p}_{i}^{k}\right)$ is finite we see that $r k(R)$ is finite.

TheORem 16. Let $R$ be a semilocal Noetherian ring of dimension one. Then $w b d(R) \leqq b d(R)<\infty$.

Proof. $R$ has only finitely many prime ideals, say $k$. Let $A$ be an ideal of $R$ and suppose that $A=\mathfrak{q}_{1} \cap \cdots \cap \mathfrak{q}_{t}$ is a primary decomposition of $A$, where $\mathfrak{q}_{i}$ is $\mathfrak{p}_{i}$-primary. Thus $0 \rightarrow R / A \rightarrow R / \mathfrak{q}_{1} \oplus \cdots \oplus$ $R / \mathfrak{q}_{t}$ is exact, and as in the proof of Theorem 15 , the Goldie dimension of $R / A$ is less than or equal to $k \cdot \max \left\{r k\left(R_{f_{i}}\right)\right\}$. Each $R_{f_{i}}$ has finite rank by Corollary 9 and an application of Lemma 14, as in the last part of the proof of Theorem 15.

Finally we mention that we have no example of a ring for which the weak bound is finite but the bound is not finite.

\section{REFERENCES}

1. M. F. Atiyah and I. G. MacDonald, Introduction to Commutative Algebra, AddisonWesley, Reading, MA, 1969. 
2. J. A. Beachy, On quasi-Artinian rings, J. London Math. Soc., (2) 3 (1971), 449-452.

3. W. Brandal, Commutative rings whose finifely generated modules decompose, Lecture Notes in Math., 723 (1979).

4. G. Cauchon, Les T-anneaux et la condition de Gabriel, C. R. Acad. Sci. Paris, 277 (Série A) (1973), 1150-1156.

5. I. S. Cohen, Commutative rings with restricted minimum condition, Duke Math. J., 17 (1950), 27-42.

6. P. Gabriel, Des catégories abéliennes, Bull. Soc. Math. France, 90 (1962), 323-448.

7. A. W. Goldie, The structure of Noetherian rings, Lecture Notes in Math., 246 (1972), 214-321.

8. I. Kaplansky, Commutative Rings, Allyn and Bacon, Boston, 1970.

9. M. D. Larsen and P. J. McCarthy, Multiplicative Theory of Ideals, Academic Press, New, York, 1971.

10. R. B. Warfield, Jr., Decomposability of finitely presented modules, Proc. Amer. Math. Soc., 25 (1970), 167-172.

Received April 18, 1980.

Northern IlLinoIS University

De KaLb, IL 60115 


\section{PACIFIC JOURNAL OF MATHEMATICS}

\section{EDITORS}

DONALD BABBITT (Managing Editor)

University of Galifornia

Los Angeles, California 90024

Hugo RossI

University of Utah

Salt Lake City, UT 84112

C. C. MOORE AND ANDREW OGG

University of California

Berkeley, CA 94720
J. DugundJI

Department of Mathematics University of Southern California Los Angeles, California 90007

R. FinN and J. Milgram Stanford University Stanford, California 94305

\section{ASSOCIATE EDITORS}

R. ARENS

E. F. BeCKenbaCh

B. H. NEUManN

F. WOLF

K. YosHIDA

\section{SUPPORTING INSTITUTIONS}

UNIVERSITY OF ARIZONA

UNIVERSITY OF BRITISH COLUMBIA

CALIFORNIA INSTITUTE OF TECHNOLOGY

UNIVERSITY OF CALIFORNIA

MONTANA STATE UNIVERSITY

UNIVERSITY OF NEVADA, RENO

NEW MEXICO STATE UNIVERSITY

OREGON STATE UNIVERSITY
UNIVERSITY OF OREGON

UNIVERSITY OF SOUTHERN CALIFONIA

STANFORD UNIVERSITY

UNIVERSITY OF HAWAII

UNIVERSITY OF TOKYO

UNIVERSITY OF UTAH

WASHINGTON STATE UNIVERSITY

UNIVERSITY OF WASHINGTON 


\section{Pacific Journal of Mathematics}

\section{Vol. 95, No. $1 \quad$ September, 1981}

John Allen Beachy and William David Blair, On rings with bounded

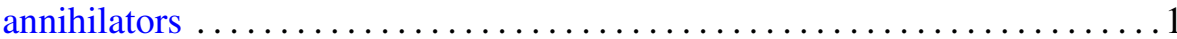

Douglas S. Bridges, A constructive look at positive linear functionals on

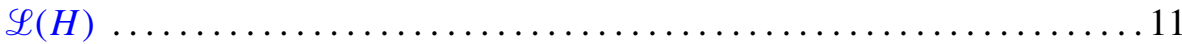

Muneo Chō and Makoto Takaguchi, Boundary points of joint numerical ranges

W. J. Cramer and William O. Ray, Solvability of nonlinear operator equations

Lester Eli Dubins and Gideon Schwarz, Equidiscontinuity of

Borsuk-Ulam functions

Maria Fragoulopoulou, Spaces of representations and enveloping 1.m.c.

*-algebras

Robert F. Geitz and J. Jerry Uhl, Jr., Vector-valued functions as families of scalar-valued functions

Ross Geoghegan, The homomorphism on fundamental group induced by a homotopy idempotent having essential fixed points

Ross Geoghegan, Splitting homotopy idempotents which have essential fixed points

Paul Jacob Koosis, Entire functions of exponential type as multipliers for

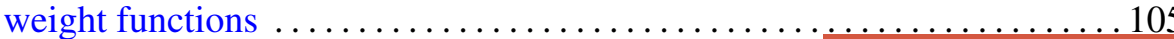

David London, Monotonicity of permanents of certain doubly stochastic matrices

Howard J. Marcum, Two results on cofibers

Giancarlo Mauceri, Zonal multipliers on the Heisenberg group

Edward Wilfred Odell, Jr. and Y. Sternfeld, A fixed point theorem in $c_{0} \quad \ldots 161$

Bernt Karsten Oksendal, Brownian motion and sets of harmonic measure zero

Andrew Douglas Pollington, The Hausdorff dimension of a set of normal numbers

Joe Repka, Base change lifting and Galois invariance ...

Gerald Suchan, Concerning the minimum of permanents on doubly stochastic circulants

Jun-ichi Tanaka, On isometries of Hardy spaces on compact abelian groups

Aaron R. Todd, Quasiregular, pseudocomplete, and Baire spaces 